\title{
PROPOSTA DE UM MODELO CONCEITUAL PARA TOMADA DE DECISÃO ENTRE CENTRALIZAR OU DESCENTRALIZAR ARMAZÉNS
}

\section{A CONCEPTUAL MODEL PROPOSAL TO DECISION MAKING ABOUT CENTRALIZATION OR DECENTRALIZATION OF STOREHOUSES}

\author{
Gilberto Marassi de Loiola Leite * E-mail: gilbertomll@gmail.com \\ Bruno Beiler* E-mail: bruno.caride.beiler@gmail.com \\ Carolinne Ferreira* E-mail: carolinne.eq@gmail.com \\ Thomas Kors* E-mail: thomaspkors@gmail.com \\ Paulo Sérgio de Arruda Ignácio* E-mail: paulo.ignacio@fca.unicamp.br \\ ${ }^{*}$ Faculdade de Ciências Aplicadas da Universidade Estadual de Campinas \\ (FCA - UNICAMP), Limeira, SP
}

\begin{abstract}
Resumo: O presente estudo tem como objetivo geral propor um modelo conceitual para a alocação de um ou múltiplos CDs (Centros de Distribuição) que considere os aspectos que influenciam o desempenho (operacional e financeiro) das operações logísticas, visando auxiliar o tomador de decisão no planejamento da distribuição que melhor atenda às características do mercado em que a empresa está inserida. O objetivo secundário é avaliar as implicações da decisão de centralizar ou não a distribuição de produtos. Trata-se de uma pesquisa indutiva e explicativa, que busca o levantamento de informações relevantes à determinação da localização de estoques de produtos acabados que, por meio de revisão bibliográfica, intenta produzir critérios de decisão a serem utilizados no método de análise multicritérios conhecido como AHP (Analytic Hierarchy Process). Em seguida, simula-se a aplicação do modelo conceitual proposto a uma hipotética distribuição de calçados em nível nacional, com estabelecimento assistemático de pesos para cada critério para um teste básico de aplicabilidade, com a realização do cálculo e da avaliação de consistência pelo software PriEsT. Para os fins a que se propõe, este trabalho apresenta importância ao reunir de forma lógica os diversos critérios, com a intenção de ser instrumento de aplicação no planejamento estratégico de distribuição das empresas com necessidades convergentes ao problema apresentado. Intenta, por fim, ser um ponto de partida para adaptações a possíveis estudos de caso.
\end{abstract}

Palavras-chave: Centro de Distribuição. Rede de Distribuição. Método de Análise Hierárquica, Tomada de decisão.

Abstract: The present study has as its main objective a conceptual model proposal to locate one or multiple DC's (Distribution Centers), considering the aspects of influence on logistics performance (operational and financial ones), aiming to aid the decision maker about the best distribution planning for the specific purposes of his marketplace. The subsidiary objective is an assessment of the implications which surround those decisions. This is an inductive and explanatory research which searches for relevant information to determine the location of goods inventories, based on literature review, producing decision criteria to be used in multi-criteria analysis method known as AHP (Analytic Hierarchy Process). Then a simulation of a practical usage of the conceptual model in a hypothetical footwear distribution in national level is given, through an unsystematic weight establishment for each criteria, as well as the respective calculation and assessment of the consistency level using the software PriEst, as an applicability test. For its intended purposes, this study is important to logically group the several criteria, willing to provide an application tool for companies' strategic distribution 
planning, in face of similar problems to presented here. Finally it attempts to be a starting point for possible future case studies and adaptations.

Keywords: Distribution Center. Distribution Network. Analytic Hierarchy Process. Decision making.

\section{INTRODUÇÃO}

As estratégias para alocação de armazéns de produtos acabados crescem em importância no campo de estudos da logística à medida que os mercados consumidores tornam-se mais dinâmicos e a concorrência para atendê-los se acirra e se sofistica. Neste cenário, há uma dupla pressão, seja para o aumento da responsividade às demandas de mercado, seja pela exigência de redução de estoques, visando, por consequência, reduzir custos (BAKER, 2004): a tomada de decisão sobre centralizar os estoques em um único CD (Centro de Distribuição) ou instalar uma rede de CDs visando regionalizar a distribuição está diretamente ligada a este trade-off.

Assim, este estudo trata do seguinte problema: é possível desenvolver um artifício teórico que auxilie o tomador de decisão a elaborar uma estratégia de alocação de armazéns que beneficie sua organização dentro das metas que lhe são típicas e próprias? A justificativa para o tema é o impacto crescente deste tipo de decisão em fatores estratégicos de distribuição: para Ballou (2006), por exemplo, a localização das instalações se torna o problema mais importante dentro do planejamento estratégico de logística em muitas empresas justamente por estabelecer condições para o gerenciamento de níveis de estoque e transporte.

Este trabalho, então, tem como objetivo geral propor um modelo conceitual para a alocação de um ou múltiplos CDs que considere os aspectos que influenciam o desempenho (operacional e financeiro) das operações logísticas, visando auxiliar o tomador de decisão no planejamento da distribuição que melhor atenda às características do mercado em que a empresa está inserida. O objetivo secundário é avaliar as implicações da decisão de centralizar ou não a distribuição de produtos.

Metodologicamente, este estudo se classifica como uma pesquisa indutiva e explicativa, que busca o levantamento de informações relevantes à determinação da localização de estoques de produtos acabados, por meio de revisão bibliográfica, a fim de produzir critérios de decisão a serem utilizados no método de análise multicritérios conhecido como AHP (Analytic Hierarchy Process). Deste modo, buscam-se obter os principais elementos de decisão, como custos, nível de serviço, tempo de atendimento, desperdícios com obsolescência de produtos, entre outros. Em seguida, simula-se a aplicação do modelo conceitual proposto a uma hipotética distribuição de calçados em nível nacional, com estabelecimento assistemático de pesos para cada critério para um teste básico de aplicabilidade, com a realização do cálculo e da avaliação de consistência pelo software PriEsT. 
É importante ressaltar que, para os fins a que este trabalho se propõe, os termos "Centro de Distribuição" (CD) e "armazém" devem ser entendidos como localidades onde produtos acabados são estocados: as diferenças entre os dois formatos e demais atividades possivelmente executadas nesses modelos de instalação fogem ao escopo deste artigo.

\section{REVISÃO DA LITERATURA}

Os custos de distribuição incidem de forma relevante sobre os custos totais da empresa, e esse é um dos motivos pelo qual a determinação de como instalar seus Centros de Distribuição em uma operação de âmbito nacional, ou mesmo global, é um problema premente. Hua et. al. (2016) destacam que um sistema de distribuição logístico eficiente é tão importante para o crescimento econômico das companhias quanto para a redução de custos desnecessários. Além dos custos, os gestores das cadeias de suprimento de cada segmento devem estar alinhados com a importância da agilidade das entregas em cada mercado: Lee e Kwon (2010) afirmam que as empresas são obrigadas a manter altos níveis de satisfação dos clientes ao mesmo tempo em que são forçadas a reduzir custos e manter as margens de lucro. A estrutura de distribuição interfere diretamente neste desempenho, e sua instalação deve também considerar aspectos qualitativos que refletem na qualidade percebida dos serviços associados à responsividade.

Apesar de haver diversos métodos para a resolução do problema de alocação de CDs para uma ou várias unidades, aparentemente não há consenso sobre a melhor forma de comparação da eficiência entre uma ou outra estratégia no âmbito acadêmico, e o tema continua relevante e amplamente discutido no meio corporativo. Wanke (2003) explica que centralizar estoques significa postergar ao máximo o transporte de produtos, para apenas movimentá-los quando o cliente final realizar o pedido. Em contrapartida, descentralizar estoques significa antecipar a movimentação por instalações intermediárias no momento presente baseando-se em previsões de vendas.

Novaes (2001) define as características destes dois tipos antagônicos de distribuição nos termos de "um para um" e "um para muitos", sendo o primeiro o transporte "lotação", onde um veículo transporta uma grande quantidade de mercadorias (completando o(s) veículo(s)) de uma fábrica ou CD para outro CD ou instalação, e o segundo caracteriza um transporte fracionado, de um único ponto de coleta para vários destinos, como lojas ou clientes.

Alguns profissionais de logística são partidários da centralização e outros defendem a descentralização, o que faz deste estudo uma importante contribuição para auxiliar os responsáveis por essa decisão, já que a solução não é simples nem unânime: Wanke (2003) pontua que nos setores alimentício, automobilístico, químico 
e petroquímico predomina a descentralização, enquanto os setores farmacêutico e eletroeletrônico são exemplos do uso de uma distribuição centralizada.

Neste contexto, é necessário entender as características de cada uma das estratégias, visando estabelecer um parâmetro de comparação que permita construir os critérios de decisão do modelo conceitual proposto. Ao projetar uma cadeia de suprimentos, as decisões de localização devem fazer parte das principais estratégias a se estruturar, pois são considerados objetivos como a minimização dos custos, a maximização das receitas e dos serviços aos clientes. Para Slack et. al. (2009) decisões de localização são, basicamente, decorrentes de fatores no lado da demanda e de fatores no lado da oferta, conforme a Tabela 1:

Tabela 1 - Fatores influenciadores da localização

\begin{tabular}{ll}
\multicolumn{1}{c}{$\begin{array}{c}\text { Fatores do lado da demanda } \\
\text { (influenciam mais os custos) }\end{array}$} & \multicolumn{1}{c}{$\begin{array}{c}\text { Fatores do lado da oferta } \\
\text { (influenciam mais os serviços / receitas) }\end{array}$} \\
\hline Custos de mão de obra & Habilidades de mão de obra \\
Custos de terra & Adequação do local \\
Custos de energia & Imagem \\
Custos de transporte & Conveniência para os clientes \\
Fatores da comunidade & \\
\hline
\end{tabular}

Fonte: Adaptado de Slack et. al. (2009)

Decisões de localização de estoque são fundamentais, pois representam altos investimentos e são muitas vezes difíceis de reverter, por exemplo, devido aos custos de interrupção serem maiores do que os benefícios da mudança (SLACK et. al., 2009). A exigência do mercado, tal como a disponibilidade buscada, é outro fator decisivo para a localização, pois é o fim a que se propõem praticamente todas as organizações, mas também impacta fortemente os custos e por isso necessita de análises de compensação entre custos de transporte e custos da manutenção do estoque, assim como a flexibilidade dos processos de fabricação também afetarão as decisões de localização ou, mais especificamente, as decisões de centralização ou descentralização dos estoques na cadeia de suprimentos (TORRES, 2003).

Baker (2004) expande o conceito do papel dos Centros de Distribuição, não apenas com a ênfase nos custos ou serviços individualmente, mas em sua adequação à estratégia de toda a cadeia de suprimentos. Desta forma, há uma importante relação (não necessariamente oposta) entre os conceitos de produção enxuta (lean concept), buscando a eliminação de desperdícios no processo e assim reduzir custos, e o conceito de produção ágil (agility), que desenvolve diferenciais de atendimento, sobretudo em mercados voláteis, objetivando incrementos no nível de serviço: em cadeias de suprimento enxutas se busca a redução dos níveis de estoque, e nas cadeias de suprimento ágeis estoques são mantidos de tal forma a poder responder rapidamente às exigências do mercado. Por esse motivo, Baker (2008) aponta que os Centros de Distribuição devem ser vistos como parte de um 
sistema mais amplo (que engloba toda a cadeia de suprimentos), tornando-se assim parte da própria estrutura competitiva da empresa.

Segundo Wanke (2011), as características que interferem no grau de centralização de estoques de produtos acabados numa cadeia de suprimentos podem ser resumidas em quatro características:

- giro do produto: Produtos com alto giro absorvem parcelas menores dos custos fixos de armazenagem e possuem riscos menores de perecibilidade e obsolescência, portanto tendem a ser indicados para descentralização (TORRES, 2003; VIEIRA, 2009; WANKE, 2011);

- tempo de atendimento: Conforme os tempos de atendimento (tempo entre colocação do pedido e o recebimento pelo cliente) aumentam, tende a ser aconselhável uma descentralização visando agilidade. Deve-se avaliar se a abertura de um novo centro de distribuição mais do que compensa o custo de oportunidade de manter o estoque em trânsito que seria gerado com a centralização (TORRES, 2003; VIEIRA, 2009; WANKE, 2011);

- nível de serviço: Similar à análise que deve ser feita para o tempo de resposta, quanto maior o nível de serviço, maior será a tendência de descentralização visando localizar os produtos mais próximos do cliente (TORRES, 2003; VIEIRA, 2009; WANKE, 2011);

- custos unitários de aquisição: Quanto maiores esses custos, maior será a tendência de centralização devido ao elevado custo de oportunidade de manter esses estoques descentralizados (TORRES, 2003; VIEIRA, 2009; WANKE, 2011).

A centralização de estoques reduz os estoques de segurança fazendo com que a empresa ganhe com a compensação da flutuação da demanda em diferentes regiões. A descentralização aumenta o risco do desbalanceamento de estoques e gastos adicionais com transferência de produtos entre os centros de distribuição (WANKE, 2011).

A mesma questão é abordada também por Chopra e Meindl (2011), que pontuam que o objetivo do planejador logístico é compreender de que forma é possível explorar a agregação de estoque sem prejudicar a disponibilidade do produto, reduzindo ao máximo o estoque de segurança para reduzir custos. Assim, eles apontam duas desvantagens principais na centralização: o aumento do tempo de resposta ao pedido do cliente e o aumento do custo de transporte ao cliente.

Christopher (1997) elenca cinco fatores criticamente importantes ao se considerar a centralização ou não de estoques de produtos acabados: prazos longos de fornecimento; tempos de trânsito extensos e não-confiáveis; opções de consolidação e desmembramento de volumes; modais de transporte e opções de 
custos; expedição de componente semimanufaturado com valor adicionado localmente.

Moura (1989), por sua vez, aponta outras características relevantes no estabelecimento ou não de um estoque centralizado, como os custos de duplicação do número de pessoal em vários armazéns, e a redução do espaço total e do custo em um ambiente centralizado, dado que o custo por metro quadrado tende a ser menor em um grande CD do que se a mesma unidade de espaço for usada em diversas localidades e edifícios. Porém, ele destaca o trade-off envolvido entre localização e frete, já que os armazéns devem ser locados entre os fornecedores e os clientes, o que implica no estabelecimento dos mesmos nas proximidades de centros de tráfego, onde o custo do terreno é alto.

De maneira geral, estas são as informações mais imediatas que a pesquisa obteve e que o tomador de decisão deve se atentar ao elaborar a estratégia logística de distribuição de seus produtos, mas pode não esgotar os pontos de influência, dada a complexidade do tema. Esse estudo intenta extrair das informações supracitadas critérios logicamente estruturados, a fim de organizá-los de forma a desenvolver um modelo conceitual de auxílio à decisão sobre a melhor estratégia diante de cada situação específica. Não se lança mão da nomenclatura dada por um ou outro autor especificamente, por diferirem na maior parte das vezes: procura-se incluir cada um dos conceitos elencados para que sejam ponderados pelo tomador de decisão que eventualmente fizer uso do modelo.

\subsection{Método AHP}

Diversas técnicas em Pesquisa Operacional vêm sendo utilizadas para auxiliar a tomada de decisões no gerenciamento da cadeia de suprimentos e logística. Uma das técnicas que tem por finalidade o estudo de problemas com diversos critérios simultaneamente é a metodologia multicritério de apoio à decisão (MCDA - Multicriteria Decision Analysis), que busca selecionar a melhor escolha dentre um conjunto de alternativas. O método mais conhecido dentro deste contexto é o de análise hierárquica (AHP - Analytic Hierarchy Process), desenvolvido por Thomas L. Saaty em 1980, que auxilia o processo de tomada de decisão dividindo o problema em níveis hierárquicos, com base no conhecimento e na experiência dos envolvidos na tomada de decisões (BELFIORE e FÁVERO, 2012).

O método AHP baseia-se na teoria generalista da medida, derivando escalas de razão de comparações pareadas discretas e contínuas. Tais comparações podem ser extraídas de medidas reais ou de uma escala fundamental, refletindo a força relativa das preferências dentro e entre os grupos de elementos da estrutura. Suas amplas aplicações em tomada de decisão multicritério contemplam planejamento, alocação de recursos e resolução de conflitos. 
Este método manipula a complexidade de um problema, decompondo-o e dividindo-o em fatores, que por sua vez também podem ser decompostos em subfatores, até que se chegue a um nível mais baixo e claro - que são as alternativas do problema. De acordo com Costa (2002), o método apresenta três etapas do pensamento analítico:

- construção de hierarquias: a estruturação em níveis hierárquicos facilita a avaliação e a compreensão do problema. Para isso, deve-se representar o problema o mais completamente possível, considerar o ambiente que o envolve, identificar as questões que contribuem para a solução e identificar os participantes relacionados com o problema. $\mathrm{O}$ primeiro nível da hierarquia deve corresponder ao objetivo geral do problema, o segundo aos critérios e o terceiro às alternativas. Com isso, tem-se uma visão geral das relações complexas inerentes ao problema e o tomador de decisão avalia com mais precisão a ordem de grandeza dos elementos de cada nível. A Figura 1 mostra o fluxograma do método:

Figura 1 - Fluxograma genérico do método AHP

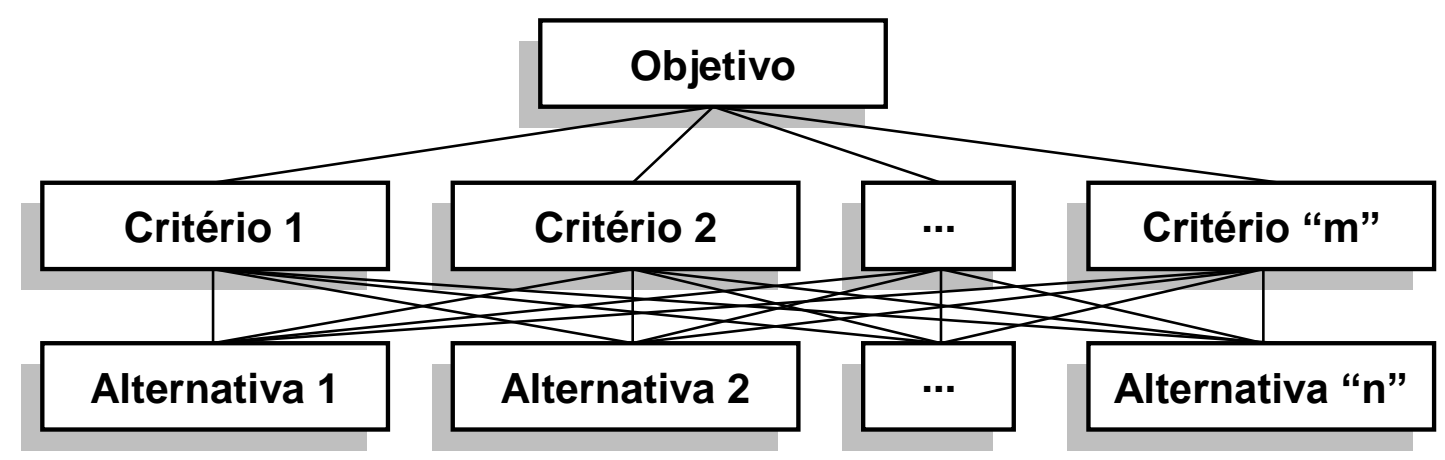

Fonte: MARINS, C. S.; SOUZA, D. O.; BARROS, M. S. (2009)

- definição de prioridades: neste estágio, faz-se necessário que o tomador de decisão perceba a relação entre os critérios, realizando julgamentos paritários: julgar par a par os elementos do mesmo nível de hierarquia, compondo assim a matriz de julgamentos $A$. Para isso, utilizase uma escala para avaliações contendo 5 pontos de avaliação e 4 pontos intermediários, a chamada Escala Fundamental de Saaty, apresentada na tabela 2: 
Tabela 2 - Escala Fundamental de Saaty

\begin{tabular}{|c|c|c|}
\hline $\begin{array}{c}\text { Escala } \\
\text { numérica }\end{array}$ & Escala verbal & Explicação \\
\hline 1 & $\begin{array}{l}\text { Ambos os elementos são de igual } \\
\text { importância. }\end{array}$ & $\begin{array}{l}\text { Ambos os elementos contribuem } \\
\text { com a propriedade de igual forma. }\end{array}$ \\
\hline 3 & $\begin{array}{l}\text { Moderada importância de um } \\
\text { elemento sobre o outro. }\end{array}$ & $\begin{array}{c}\text { A experiência e a opinião favorecem } \\
\text { um elemento sobre o outro. }\end{array}$ \\
\hline 5 & $\begin{array}{l}\text { Forte importância de um elemento } \\
\text { sobre o outro. }\end{array}$ & $\begin{array}{l}\text { Um elemento é fortemente } \\
\text { favorecido. }\end{array}$ \\
\hline 7 & $\begin{array}{l}\text { Importância muito forte de um } \\
\text { elemento sobre o outro. }\end{array}$ & $\begin{array}{l}\text { Um elemento é muito fortemente } \\
\text { favorecido sobre o outro. }\end{array}$ \\
\hline 9 & $\begin{array}{l}\text { Extrema importância de um } \\
\text { elemento sobre o outro. }\end{array}$ & $\begin{array}{l}\text { Um elemento é favorecido pelo } \\
\text { menos com uma ordem de } \\
\text { magnitude de diferença. }\end{array}$ \\
\hline $2,4,6,8$ & $\begin{array}{c}\text { Valores intermediários entre as } \\
\text { opiniões adjacentes. }\end{array}$ & $\begin{array}{l}\text { Usados como valores de consenso } \\
\text { entre as opiniões. }\end{array}$ \\
\hline $\begin{array}{l}\text { Incremento } \\
0,1\end{array}$ & $\begin{array}{c}\text { Valores intermediários na } \\
\text { graduação mais fina de } 0,1 \text {. }\end{array}$ & $\begin{array}{l}\text { Usados para graduações mais finas } \\
\text { das opiniões. }\end{array}$ \\
\hline
\end{tabular}

Fonte: Roche (2004, p.6)

A quantidade de comparações necessárias para compor a matriz de decisão, estabelecida como uma matriz $A=\left[a_{i j}\right]$ de ordem $n \times n$, é definida por:

$$
\frac{n(n-1)}{2}
$$

em que $n$ corresponde ao número de elementos que pertence à matriz. Além disso, a construção da mesma deve obedecer às seguintes regras:

$$
\begin{gathered}
\boldsymbol{a}_{i j}>0 \text { (matriz positiva) } \\
\boldsymbol{a}_{i j}=\mathbf{1} \text {, se } i=j \\
\boldsymbol{a}_{i j}=\frac{1}{a_{i j}} \text { (recíproca) } \\
\boldsymbol{a}_{i k}=\boldsymbol{a}_{i j} \times \boldsymbol{a}_{j k} \text { (matriz consistente) }
\end{gathered}
$$

- consistência lógica: Saaty (2000) destaca que o ser humano tem a habilidade de estabelecer relações entre objetos ou ideias de forma que elas sejam coerentes, tal que estas se relacionem bem entre si e suas relações apresentem consistência. Portanto, após a construção da matriz de decisão $A$, calcula-se a razão de consistência dos julgamentos (RC), utilizando-se a equação 6 :

$$
R C=\frac{I C}{I R}
$$


na qual IC = índice de consistência, definido pela equação 7 , em que Amax é o maior autovalor da matriz de decisão, e $\mathbf{I R}=$ índice de consistência randômico, obtido para a matriz recíproca de ordem $n$, cujos elementos são não-negativos. Saaty propôs índices aleatórios para matrizes conforme a sua ordem, conforme exibido na tabela 3.

$$
\frac{\lambda \max -n}{n-1}
$$

Tabela 3 - Índices randômicos

\begin{tabular}{c|ccccccccccccccc}
$\mathbf{n}$ & $\mathbf{1}$ & $\mathbf{2}$ & $\mathbf{3}$ & $\mathbf{4}$ & $\mathbf{5}$ & $\mathbf{6}$ & $\mathbf{7}$ & $\mathbf{8}$ & $\mathbf{9}$ & $\mathbf{1 0}$ & $\mathbf{1 1}$ & $\mathbf{1 2}$ & $\mathbf{1 3}$ & $\mathbf{1 4}$ & $\mathbf{1 5}$ \\
IR & 0.00 & 0.00 & 0.58 & 0.90 & 1.12 & 1.24 & 1.32 & 1.41 & 1.45 & 1.49 & 1.51 & 1.48 & 1.56 & 1.57 & 1.59 \\
\hline
\end{tabular}
Fonte: SAATY, T. L. (1991)

A matriz é considerada consistente para valores de $\mathrm{RC}$ menores que 0,1. Para valores maiores, o tomador de decisão deve reconsiderar a sua avaliação.

Em suma, o processo analítico hierárquico utiliza comparações aos pares e então calcula as ponderações dos fatores e avaliações, e também é importante que seja calculada a razão de consistência para que o processo seja aceito, caso contrário, as respostas devem ser revistas. O tomador de decisão inicia o processo fazendo o detalhamento da hierarquia da decisão que representa uma visão geral, em seguida identifica os fatores que serão considerados, assim como as diversas alternativas no processo decisório. Na sequência, são feitas comparações aos pares, que são realizadas na forma de matrizes com uma escala de comparação de 1 a 9 (de igualmente preferido a extremamente preferido), resultando na determinação dos pesos dos fatores e na avaliação dos mesmos. Todo este processo retira um pouco da subjetividade e permite uma confiança maior na determinação de pesos e na avaliação (RENDER et. al., 2010).

É importante ter em vista que, como o método AHP tem como objetivo estruturar o problema em níveis hierárquicos, de acordo com os objetivos, critérios, subcritérios e, por fim, as alternativas, ele deve ser compreendido como um método de análise, e não como se promovesse um direcionamento direto de qual política, no caso, de alocação de centros de distribuição, deve ser adotada: ele oferece instruções de como tomar esta decisão. Kengpol (2002), em sua particular interpretação do método, esclarece que o nível zero da hierarquia é o seu macro objetivo principal, e é a partir dele que todos os outros elementos de análise se seguirão. O nível hierárquico um na análise consiste nos critérios ou fatores a serem avaliados, e estes são os principais elementos que serão avaliados para as suas alternativas de tomada de decisão. No nível hierárquico dois, tais critérios/fatores são detalhados (atribuindo-se aí seus pesos na tomada de decisão), sendo neste nível que os atributos qualitativos, em alguma medida, se tornarão quantitativos. Ainda segundo Kengpol (2002), é especificado que tais pesos ou comparações devem respeitar certos axiomas: 
- reciprocidade: este axioma dá a ideia intuitiva de que, se a alternativa $A$ é $n$ vezes preferível à alternativa $B$, logo a alternativa $B$ é $1 / n$ vezes preferível a A;

- comparabilidade: este axioma sugere que comparações só têm algum sentido se os elementos são, de fato, comparáveis;

- dependência: este axioma permite analisar conjuntos de elementos que dizem respeito a outro elemento num nível hierárquico superior, ou seja, comparações num nível inferior dependem dos elementos do seu respectivo nível superior;

- expectativas: este axioma implica que qualquer mudança na estrutura hierárquica demandará novas avaliações de preferência (pesos) para a nova hierarquia.

\section{MÉTODO}

No que concerne ao método de pesquisa, este estudo pode ser classificado nos seguintes aspectos: quanto à sua natureza, esta pesquisa procura gerar conhecimentos para aplicação prática dirigidos à solução do problema dado, e por isso se caracteriza pela tentativa de uma compreensão dos significados e quesitos situacionais apresentados nos critérios que compõem o modelo conceitual: a análise de dados, portanto, tende a ser indutiva (SILVA; MENEZES, 2000); do ponto de vista de seus objetivos, segundo a classificação apresentada por Gil (1991), trata-se de uma pesquisa explicativa, já que visa identificar os fatores que determinam ou contribuem para a decisão de centralizar a distribuição ou não. Por fim, quanto à sua estratégia de pesquisa, aproxima-se de uma pesquisa experimental nos moldes propostos por Yin (2001), por buscar as variáveis que influenciam o objeto de estudo (a distribuição física de materiais acabados), e suas implicações em determinado cenário.

A proposta de utilização do AHP é justificada por se tratar de um método amplo e que possibilita a inserção de aspectos quantitativos e qualitativos para a tomada de decisão, o que se adequa ao presente problema, pois a centralização ou descentralização de CDs leva em consideração critérios de natureza diversa: custos de várias origens, motivos de razão estratégica, melhoria de desempenho da distribuição, redução dos prazos de entrega, etc. Ao se estabelecer todos os níveis hierárquicos, os dados são cruzados com as suas alternativas de decisão e, desta forma, o processo será capaz de auxiliar o planejador logístico no problema proposto no presente trabalho, fornecendo, neste sentido, os elementos / características que serão avaliados e contrapostos um a um entre as possíveis alternativas. O resultado prático esperado deve ser um escalonamento valorado de cada uma das suas 
alternativas, às quais o tomador de decisão escolherá o caminho que melhor se enquadre ao seu macro objetivo.

Com a finalidade de exemplificar o funcionamento do modelo, este foi aplicado de forma simulada em uma hipotética indústria com cobertura nacional de distribuição. As preferências são estabelecidas por meio de reunião de consenso entre os autores, de forma assistemática, com o único intento de testar a aplicabilidade do modelo conceitual aqui elaborado.

\subsection{Modelagem}

Através de levantamento bibliográfico diante do problema apontado, define-se um quadro, em quatro níveis, onde estão inseridos os critérios e os subcritérios que levam à determinação da alternativa mais indicada a cada caso, conforme representado a seguir:

Figura 2 - Hierarquia de decisão sobre a centralização ou não de armazéns de produtos acabados

Nível 0: Objetivo

Determinar a centralização ou descentralização de armazéns de produtos acabados

Nível 1: Critérios

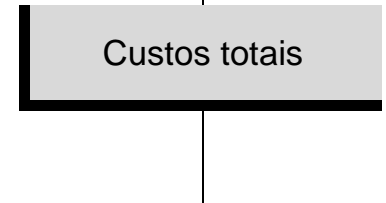

Nível 2:

Detalhamento dos

Critérios

Nível 3:

Alternativas
Custos de aquisição predial

Incentivos fiscais

Estoque de segurança / manut. de inventário Custos administrativos e de pessoal

Riscos inerentes à localização

Desbalanceamento dos estoques

Custos de frete
Tempo de resposta ao pedido

Perecibilidade / obsolescência

Giro do produto

Tempo de trânsito / disponib. de frota

Risco de ruptura do abastecimento

Sensibilidade do mercado à prazo

Logística reversa 
Fonte: Elaboração própria

Os critérios estabelecidos na hierarquia apresentada na figura 2 é a síntese dos elementos estratégicos mais destacados pelos autores pesquisados, aplicada ao método de análise AHP, e representa o cerne do presente trabalho e a concepção do modelo conceitual proposto. Basicamente, a determinação da melhor estratégia para a distribuição de produtos acabados, que redunda na escolha entre as alternativas de centralizar a distribuição ou lançar mão de uma rede de CDs, leva em consideração dois macrocritérios: custo e tempo de resposta. Porém, esses critérios apresentam uma série de desdobramentos que são de importante consideração e que refinam a compreensão de seu alcance e especificidade:

- custos de aquisição predial: são todos os custos envolvidos na aquisição do(s) $\mathrm{CD}(\mathrm{s})$. Como operações centralizadas envolve a aquisição de armazéns de maior metragem cúbica, o custo tende a ser diluído em virtude do aumento do poder de barganha na compra. Porém, como uma rede pode se estender por localidades com custos de imóveis distintos por características regionais, a diferença pode ocorrer inversamente (TORRES, 2003; VIEIRA, 2009; WANKE, 2011);

- incentivos fiscais: segundo Silva (2007), as unidades federativas no Brasil, em virtude de disputas entre os Estados, expedem unilateralmente leis, decretos e atos administrativos para outorgar vantagens fiscais, financeiras, creditícias e operacionais às empresas que ali se instalarem. Desta forma, a localização do CD central pode trazer benefícios ao total da operação ou, quando isso não for possível por razões de outra natureza, a rede de CDs pode trazer benefícios parciais relacionados às unidades que se instalaram em regiões onde o incentivo pode ser concedido;

- estoque de segurança / manutenção de inventário: uma operação descentralizada tende a manter estoques de segurança totais (ou seja, o somatório de todos os estoques de segurança regionais) maiores do que o que seria demandado em um único CD. Além disso, ou talvez por consequência desta característica, os custos de manutenção de inventário também são maiores em distribuições que utilizam uma rede de CDs (WANKE, 2011; CHOPRA e MEINDL, 2011);

- custos administrativos e de pessoal: os custos administrativos são majorados em uma configuração de rede de CDs, tal como o custo de pessoal, uma vez que algumas tarefas e funções que são executadas de forma específica em uma única localidade, são replicadas para cada unidade: pode-se dizer que a estrutura centralizada é mais enxuta nesses aspectos. Custos de mão de obra também dependem da região em que o CD é instalado, por características do mercado de trabalho local, e custos 
extras de capacitação e treinamento também podem ocorrer (MOURA, 1989);

- riscos inerentes à localização: é de se esperar que cada região esteja sujeita aos riscos que the são próprios, como os relacionados à segurança, ausência de mão de obra, incidências de desastres naturais, qualidade da infraestrutura pública, etc. Em uma rede de distribuição esse risco é diluído, mas justamente por isso aumentam as chances de ocorrência em algum ponto específico. Centralizando a operação o risco diminui, mas os efeitos de sua ocorrência tendem a ser mais danosos (SCHOENHERR e TUMMALA, 2011);

- desbalanceamento dos estoques: caso não haja controles eficientes e integrados, aumenta-se o risco de desbalanceamento de estoques em uma operação envolvendo várias unidades de distribuição. Cabe ao tomador de decisão analisar se diante de sua organização essa característica pode representar um problema (WANKE, 2011; CHOPRA e MEINDL, 2011);

- custos de frete: de forma geral, uma distribuição com origem em um único ponto gera custos de fretes fracionados de distribuição nacional, que normalmente são executados por transportadores terceirizados, pela necessidade de uma malha de distribuição mais capilarizada. Já para as operações regionalizadas os custos são compostos por fretes consolidados de transferência (cargas fechadas, que podem ser feitas até mesmo com frota própria) até os CDs, e a distribuição local a partir deste ponto. É preciso analisar qual composição é mais econômica, mas muito comumente a operação em rede possui custo de transporte menor (CHOPRA e MEINDL, 2011; CHRISTOPHER, 1997). Além disso, Huang et al (2012) incluem os custos de transporte dos fornecedores aos CDs como fator relevante;

- perecibilidade / obsolescência: se o produto, por características de sua própria composição ou mercado, tiver um reduzido período de durabilidade, seja por perecibilidade ou por obsolescência, é altamente indicado que sua disponibilidade seja mais ágil, o que é melhor garantido por meio de uma distribuição regionalizada (BAKER, 2004);

- giro do produto: produtos de maior giro reduzem custos de armazenagem, mas demandam disponibilidade frequente e constante, e por isso uma estrutura de rede de distribuição é mais indicada para produtos com essas características, já que reduz a possibilidade de falta de produto pela proximidade com o seu mercado consumidor (TORRES, 2003; VIEIRA, 2009; WANKE, 2011);

- tempo de trânsito / disponibilidade de frota: operações centralizadas podem apresentar maior tempo de trânsito se a área de cobertura de 
clientes for bastante dispersa. No entanto, pode não haver na localidade específica onde um $C D$ regional foi estabelecido uma estrutura de transporte direto para esta distribuição local (por falta de fluxo), o que pode gerar aumentos de prazo em virtude da exigência de transbordos em grandes centros, ou a necessidade de despender frotas próprias para 0 atendimento eficiente (BALLOU, 2006; BOWERSOX e CLOSS, 2009);

- risco de ruptura do abastecimento: como toda diluição de risco, o estabelecimento de vários CDs apresenta dois vieses: por um lado, o risco de uma ruptura no abastecimento é dividido entre os CDs, possibilitando até mesmo estratégias operacionais de atendimento entre as unidades no caso de alguma falha pontual e assim diminuindo o dano; por outro lado, a possibilidade de sua ocorrência é multiplicada pelo número de unidades, o que demanda ponderação sobre este aspecto (SCHOENHERR e TUMMALA, 2011);

- sensibilidade do mercado à prazo: há mercados em que a sensibilidade ao prazo de entrega é maior do que outros, como mercados que não administram estoques no varejo, ou com tipo de venda associada ao serviço, como autopeças, informática, etc. Outros mercados consideram a agilidade importante, porém esse não é fator decisivo ou gerador de custo, como confecção, calçados, livros, etc., onde o tempo de exposição na gôndola ou vitrine é relevante, mas não se sente os impactos da indisponibilidade com tanta intensidade (ou não há a cultura de medi-los ou considerá-los como críticos). Desta forma, é preciso analisar se a quantidade de clientes em regiões mais afastadas e suas exigências de prazo justifica a proximidade física dos estoques a esses mercados (BAKER, 2004, 2008);

- logística reversa: uma distribuição em rede aumenta sobremaneira a eficiência de operações de logística reversa, pela proximidade da estrutura com seus mercados consumidores, que facilita a coleta e pode reduzir fretes, se a intenção do retorno é um descarte específico, por exemplo. Oliveira (2016) destaca a grande importância socioeconômica deste tipo de operação, e a crescente busca por ações de sustentabilidade que lançam mão deste fluxo inverso.

Esses critérios representam as variáveis mais importantes para a decisão sobre o problema proposto, como encontrados na literatura pesquisada. Estender o número de critérios pode tornar a análise mais complexa, por aumentar a quantidade de entrelaçamento das comparações por parte do tomador de decisão, mas é plenamente possível, diante da diversidade das situações práticas. Como destaca Colin (2015), sugere-se que o número de fatores em cada um dos níveis do método AHP não deva ultrapassar nove, mas esta é uma recomendação que não precisa ser 
seguida rigorosamente caso haja uma justificativa. Como este trabalho busca um modelo conceitual de ampla aplicação e generalidade, o detalhamento dos critérios de nível 2 ultrapassa esse limite, mas como é facultado ao tomador de decisão a adaptação do mesmo à sua própria realidade, é aceitável ou mesmo esperado que critérios que não se apliquem a um exemplo específico sejam suprimidos. Com o objetivo de testar sua aplicabilidade, desenvolve-se na sequência uma simulação hipotética.

\section{APLICAÇÃo}

A seguir, apresentam-se as matrizes com os pesos aplicados a cada critério e em cada nível, para uma visão completa do exemplo hipotético:

Para a realização do cálculo que determina a decisão e também para apurar os índices de consistência, utilizou-se o software PriEsT (Priority Estimation Tool), ferramenta de uso livre e gratuito desenvolvida na Universidade de Manchester. O programa, além de auxiliar na organização dos dados, aponta as possíveis inconsistências e elabora gráficos e análises de sensibilidade que facilitam a compreensão dos resultados e das relações ente os critérios em cada nível. 
Tabela 4 - Definição de prioridades: aplicação de pesos ao modelo hierárquico

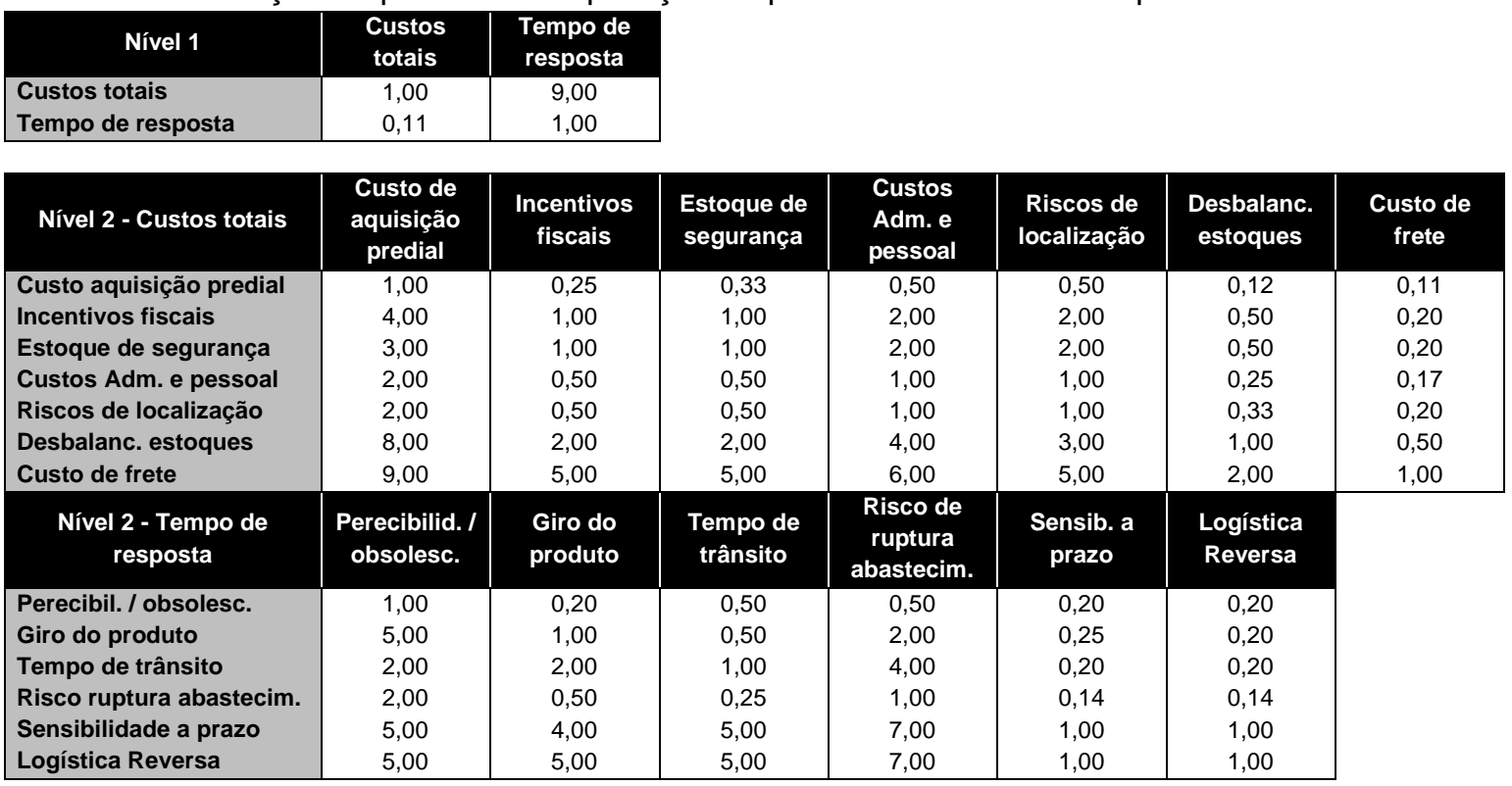

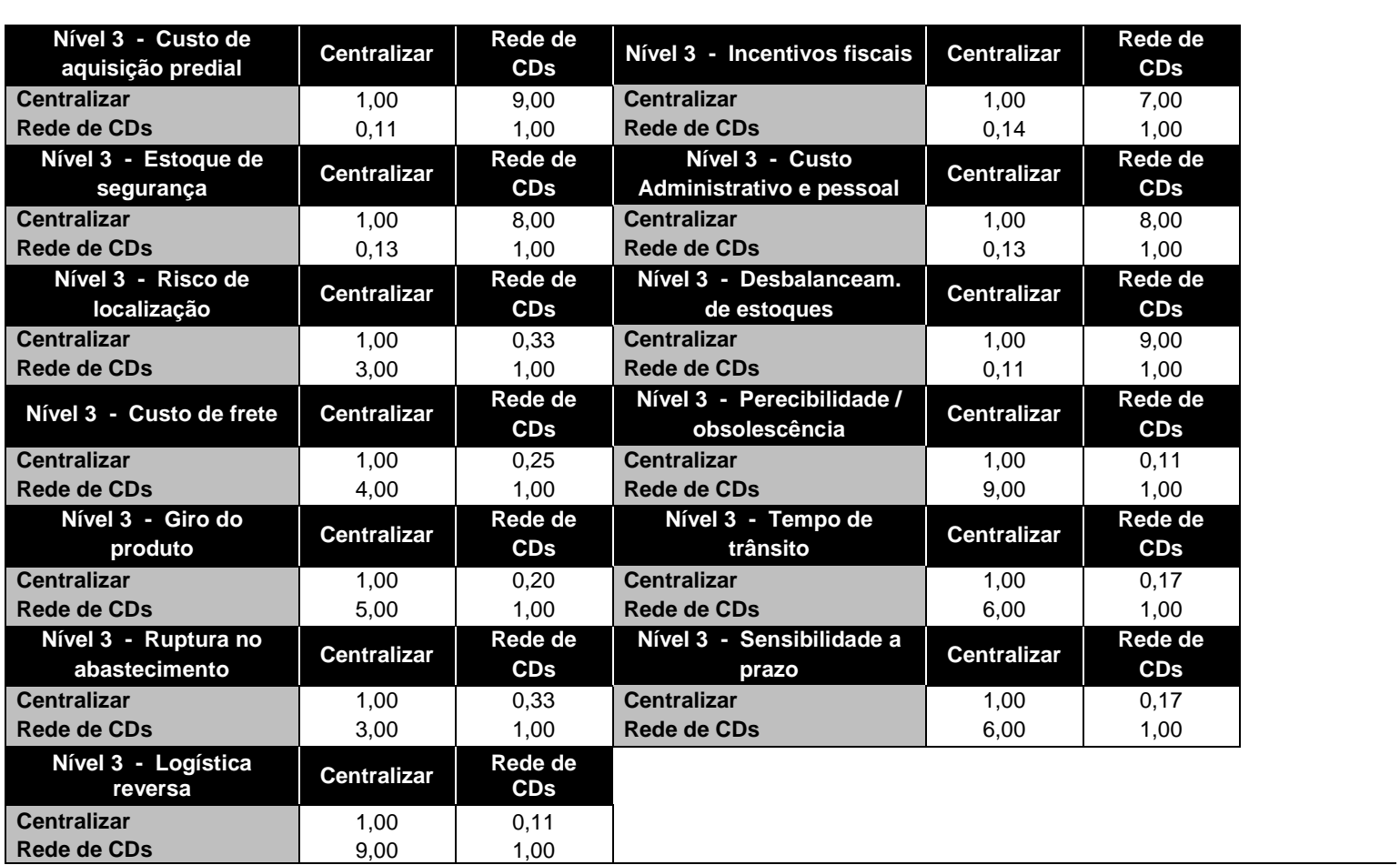

Fonte: Elaboração própria 
Tabela 5 - Indicadores de consistência gerados pelo PriesT após a aplicação dos pesos

\begin{tabular}{|c|c|c|c|}
\hline Indicador & Tradução (livre) & Custos totais & $\begin{array}{l}\text { Tempo de } \\
\text { resposta }\end{array}$ \\
\hline Dissonance $(\Psi)$ & Dissonância & 0 & 0,083 \\
\hline Congruence $(\Theta)$ & Congruência & 0,231 & 0,704 \\
\hline Three-way Cycles (L) & $\begin{array}{l}\text { Julgamentos triangularmente } \\
\text { inconsistentes }\end{array}$ & 0 & 0 \\
\hline Consistency Ratio (CR) & Razão de Consistência & 0,01 & 0,074 \\
\hline $\begin{array}{l}\text { Consistency Measure } \\
(\mathrm{CM})\end{array}$ & Medida de Consistência & 0,55 & 0,8 \\
\hline
\end{tabular}

Fonte: Elaboração própria

$\mathrm{Na}$ tabela 5 são exibidos os indicadores de consistência obtidos após a inserção no PriEsT dos pesos estabelecidos na tabela 4. Para os fins deste estudo, não se analisou os indicadores de dissonância e congruência, que são métodos auxiliares de análise da consistência do método, tal como não se lançou mão da medida de consistência, que procura suprir as desvantagens da análise da razão de consistência, sobretudo quando a mesma se encontra no limite formal (SIRAJ, 2011), porém é relevante se observar que o programa não identificou nenhum julgamento triangularmente inconsistente (do tipo: $A$ é preferível a $B$, que é preferível a $C$, que é preferível a $A$ ) nos dois critérios. Também são dignas de nota as Razões de Consistência: os pesos estabelecidos para a tomada de decisão apresentaram Razão de Consistência de 0,01 para os custos totais e de 0,074 para o tempo de resposta; como a Razão de Consistência indicada para uma análise consistente deve ser limitada a 0,1 , os resultados obtidos mostraram-se satisfatórios, pois não houve extrapolação deste índice em nenhum dos critérios.

Além disso, o PriEsT apresenta os percentuais de preferência de cada subcritério em relação ao critério principal. Pelo método Eigenvector ( $E V$ : Autovetor, em tradução livre), os resultados apresentados para este caso em particular são os exibidos na tabela 6 : 
Tabela 6 - Percentuais de preferência dos subcritérios em relação ao critério de nível 1.

\begin{tabular}{lc}
\multicolumn{1}{c}{ Custos totais } & Preferência (\%) \\
\hline Custos de aquisição predial & 3,3 \\
Incentivos fiscais & 11,1 \\
Estoque de segurança & 10,6 \\
Custos Administrativos e de pessoal & 5,9 \\
Riscos de localização & 6,4 \\
Desbalanceamento de estoques & 21,8 \\
Custo de frete & 40,9 \\
\hline \multicolumn{1}{c}{ Tempo de resposta } & Preferência (\%) \\
\hline Perecibilidade / obsolescência & 4,3 \\
Giro do produto & 9,7 \\
Tempo de trânsito & 11,3 \\
Risco de ruptura no abastecimento & 4,8 \\
Sensibilidade a prazo & 34,2 \\
Logística Reversa & 35,7 \\
\hline Fonte: Elaboração própria
\end{tabular}

Por fim, o programa apresentou que, com uma prioridade de 0,524 contra 0,476 , é indicada a centralização do estoque de produtos acabados em um único CD para os pesos fornecidos.

\subsection{Análise}

Uma vez exemplificada a aplicação do modelo conceitual, é necessário fazer uma ressalva em relação ao elevado número de comparações que o mesmo exige do tomador de decisão: para a realização deste método, foram feitas 141 comparações. Trata-se de uma característica inerente ao próprio método AHP - que por seu procedimento de comparações par a par cresce em complexidade à medida que o número de critérios e alternativas aumenta -, e consequência da própria generalidade do modelo conceitual proposto: por seu caráter genérico, é lógico supor que adaptações a cada caso particular podem reduzir o número de critérios, por não haver relevância para determinada instância ou por haver equivalência ou interdependência entre os critérios. Saaty (2005) desenvolveu um método alternativo em rede para tratar decisões cujos critérios apresentam interdependência, o ANP (Analytic Network Process), mas como a interdependência pode não existir, ou mesmo não ser óbvia em cada aplicação particular, mesmo havendo critérios cuja elicitação envolve a mesma unidade de medição (como o dinheiro, no caso de critérios ligados aos custos), o método AHP apresenta vantagens na estruturação de um modelo conceitual de amplo espectro. Além disso, o fato do método AHP ser mais longevo o torna mais difundido, ampliando as alternativas de ferramentas onde 
o modelo conceitual aqui proposto pode ser aplicado e multiplicando os casos de utilização bem sucedidos: Saaty (2005) afirma que há muitos exemplos em que a aplicação do AHP se adéqua bem a problemas da vida real, em vários segmentos e em diversos países. Baseando-se nesses diferenciais que o estudo lança mão do método em particular, mas é importante que o tomador de decisão tenha ciência das fragilidades inerentes ao mesmo, já que se trata apenas de um balizador da escolha.

\section{CONSIDERAÇÕES FINAIS}

O principal resultado deste trabalho é a obtenção dos parâmetros de comparação qualitativos e quantitativos entre uma estrutura de distribuição centralizada ou descentralizada para produtos acabados, para que o tomador de decisão, no momento de estabelecer sua estrutura, possa vislumbrar os principais fatores de influência (mesmo os de naturezas distintas), ponderar sobre os critérios e obter a alternativa que mais se adeque à realidade em que está inserido.

Como o objetivo geral é desenvolver um modelo conceitual, e não a aplicação do mesmo a casos específicos, o estudo se restringe a apresentar e detalhar os significados de seus critérios após revisão bibliográfica, configurar o modelo conforme estrutura estabelecida pelo método AHP e exibir sua aplicabilidade por meio de uma simulação, calcada em pesos estabelecidos de forma assistemática e, justamente por isso, com a única pretensão de oferecer um exemplo de seu uso. Neste intento, o trabalho obteve êxito, mas a utilização desta orientação a outras necessidades particulares e seus desdobramentos é relegada às possíveis futuras aplicações, seja por parte de novos estudos acadêmicos que façam uso do presente artigo, seja por eventual interesse em segui-lo para objetivos práticos corporativos.

Com sua natureza quali-quantitativa e, em sua aplicação, subjetiva, o modelo conceitual deve ser encarado como auxiliar no processo decisório, sem pretensões à otimalidade de seus resultados. Isso porque a realidade de cada mercado, de cada segmento de mercado e até mesmo a estratégia de cada empresa individualmente irá estabelecer os pesos de relevância para que o modelo oriente o tomador de decisão à estratégia de distribuição que melhor the cabe, pesos esses oriundos exclusivamente da visão e experiência do planejador logístico.

\section{REFERÊNCIAS}

BALLOU, R. H. Gerenciamento da cadeia de suprimentos: logística empresarial. São Paulo: Bookman, 2006.

BAKER, P. Aligning distribution center operations to supply chain strategy. International Journal of Logistics Management, Bingley, v. 15, p.111-123, 2004.

http://dx.doi.org/10.1108/09574090410700266 
BAKER, P. The design and operation of distribution centers within agile supply chains. International Journal of Production Economics, Amsterdam, v. 111, p.27-41, 2008. http://dx.doi.org/10.1016/j.ijpe.2006.09.019

BELFIORE, P.; FÁVERO, L. P. Pesquisa operacional para cursos de Administração, Contabilidade e Economia. Rio de Janeiro: Elsevier Brasil, 2012.

BOWERSOX, D. J.; CLOSS, D. J. Logística empresarial: o processo de integração da cadeia de suprimento. São Paulo: Atlas, 2009.

COLIN, E. C. Pesquisa operacional: 170 aplicações em estratégia, finanças, logística, produção, marketing e vendas. Rio de Janeiro: LTC, 2015.

COSTA, H. G. Introdução ao método de análise hierárquica: análise multicritério no auxílio à decisão. Niteroi: H.G.C., 2002.

COUNCIL OF SUPPLY CHAIN MANAGEMENT PROFESSIONALS. CSCMP Supply Chain Management Definitions and Glossary. Disponível em <https://cscmp.org/about-us/supplychain-management-definitions >. Acesso em 17/09/2015.

CHOPRA, S.; MEINDL, P. Gestão da cadeia de suprimentos: estratégia, planejamento e operações. 4. ed. São Paulo: Pearson-Prentice Hall. 2011.

CHRISTOPHER, M. Logística e gerenciamento da cadeia de suprimentos: estratégias para a redução de custos e melhoria dos serviços. São Paulo: Pioneira, 1999.

DOS SANTOS, R. F.; VIAGI, A. F. Uso do método AHP (Analytic Hierarchy Process) para otimizar a cadeia de suprimentos durante o desenvolvimento integrado de produtos. In: SIMPÓSIO DE ADMINISTRAÇÃO DA PRODUÇÃO, LOGÍSTICA E OPERAÇÕES INTERNACIONAIS. 12, 2009, São Paulo. Anais... São Paulo, 2009.

GIL, A. C. Como elaborar projetos de pesquisa. São Paulo: Atlas, 1991.

HUA, Xiang. HU, Xiao. YUAN, Wuwei. Research optimization on logistics distribution center location based on adaptive particle swarm algorithm. Optik, ed. 127, p. 8443-8450, 2016. http://dx.doi.org/10.1016/j.ijleo.2016.06.032

HUANG, R.; MENEZES, M. B. C.; KIM, S. The impact of cost uncertainty on the location of a distribution center. European Journal of Operational Research, ed. 218, p.401-407, 2012. http://dx.doi.org/10.1016/i.ejor.2011.11.016

KENGPOL, Athakorn. Design of a decision support system to evaluate the investment in a new distribution centre. King Mongkut's Institute of Technology North Bangkok, Thailand. Elsevier, 2002. 
LAMBERT, D. M.; STOCK, J. R.; VANTINE, J. G. Administração estratégica da logística. São Paulo: Vantine Consultoria, 1998.

LEE, Young Hoon. KWON, Soon Geol. The hybrid planning algorithm for the distribution center operation using tabu search and decomposed optimization. Expert Systems with Applications, ed. 37, p. 3094-3103, 2010. http://dx.doi.org/10.1016/j.eswa.2009.09.020

MARINS, C. S.; SOUZA, D. O.; BARROS, M. S. O uso do método de análise hierárquica (AHP) na tomada de decisões gerenciais - um estudo de caso. In: SIMPÓSIO BRASILEIRO DE PESQUISA OPERACIONAL. 41, 2009, Bahia. Anais... Bahia, 2009.

MOURA, R. A. Logística: suprimentos, armazenagem, distribuição física. São Paulo: Instituto de Movimentação e Armazenagem de Materiais (IMAM), 1989.

NOVAES, A. G. Logística e gerenciamento da cadeia de distribuição: estratégia, operação e avaliação. Rio de Janeiro: Campus, 2001.

OLIVEIRA, U. R.; MARINS, F. A. S.; MUNIZ JUNIOR, J. Logística reversa e identificação de produtos: revisão teórica para indústria eletroeletrônica. Revista Produção Online, Florianópolis, SC, v. 16, n. 2, p. 633-677, abr./jun. 2016. http://dx.doi.org/10.14488/16761901.v16i2.2049

PORTER, M. E. Vantagem competitiva: criando e sustentando um desempenho superior. Rio de Janeiro: Elsevier, 1989.

PRIORITY ESTIMATION TOOL - PriEsT: Disponível em $<$ http://sourceforge.net/projects/priority/>. Acesso em 20/11/2015.

PRODANOV, C. C.; DE FREITAS, E. C. Metodologia do Trabalho Científico: Métodos e Técnicas da Pesquisa e do Trabalho Acadêmico. 2ª Edição. Rio Grande do Sul: Editora Feevale, 2013.

RENDER, B.; STAIR JÚNIOR, R. M.; HANNA, M. E. Análise quantitativa para administração: com Excel e POM-QM para Windows. 10. ed. Porto Alegre: Bookman, 2010.

SAATY, R. W. The analytic hierarchy process - what it is and how it is used. Math Modelling, v. 9, n. 3-5, p. 161-176, 1987. http://dx.doi.org/10.1016/0270-0255(87)90473-8

SAATY, T. L. How to make a decision: The Analytic Hierarchy Process. European Journal Of Operational Research, ed. 48, p. 9-26, 1990. http://dx.doi.org/10.1016/03772217(90)90057-I

SAATY, T. L. Método de análise hierárquica. São Paulo: Makron Books, 1991. 
SAATY, T. L. The Analytic Hierarchy and Analytic Network Processes for the measurement of intangible criteria and for decision-making. Multiple Criteria Decision Analysis. State of the Art Surveys, Cap. 9. Nova lorque: Springer, 2005.

SCHOENHERR, T.; TUMMALA, R.Assessing and managing risks using the Supply Chain Risk Management Process (SCRMP). Supply Chain Management: An International Journal, v. 16, n. 6, p. 474-483, 2011. http://dx.doi.org/10.1108/13598541111171165

SHANK, J.K.; GOVINDARAJAN, V. Strategic cost management: the new tool for competitive advantage. New York: The Free Press, 1993.

SILVA, E. L.; MENEZES, E. M. Metodologia da pesquisa e elaboração de dissertação. 2. ed. Florianópolis: UFSC, 2000.

SILVA, M. B. Otimização de redes de distribuição física considerando incentivo fiscal baseado do crédito presumido de ICMS. Dissertação (mestrado). Escola Politécnica da Universidade de São Paulo, São Paulo, 2007.

SIRAJ, S. Preference elicitation from pairwise comparisons in multi-criteria decision making. Tese (doutorado). University of Manchester. Manchester, 2011.

SIRAJ, S.; MIKHAILOV, L.; KEANE, J. A. PriEsT: an interactive decision support tool to estimate priorities from pairwise comparison judgments. International Transactions in Operational Research, v. 22, ed. 2, p. 217-235, março de 2015.

http://dx.doi.org/10.1111/itor.12054

SLACK, N.; CHAMBERS, S.; JOHNSTON, R. Administração da produção. 3. ed. São Paulo: Atlas, 2009.

TORRES, M. D. C. G. Centralizar ou descentralizar? Entendendo as decisões na gestão de estoques. 2003. 47 f. Monografia (Especialização) - Curso de Logística Empresarial, cap. 6. Universidade Cândido Mendes, Rio de Janeiro, 2003.

VIEIRA, H. F. Gestão de estoques e operações industriais. Curitiba: IESDE, 2009.

WANKE, P. Estratégia logística: conceitos, implicações e análise da realidade brasileira. In: FIGUEIREDO, K. F.; FLEURY, P. F.; WANKE, P. (Org.). Logística e gerenciamento da cadeia de suprimentos: planejamento do fluxo de produtos e dos recursos. São Paulo: Atlas, 2003.

WANKE, P. Impacto das características do negócio sobre as decisões logísticas e a organização do fluxo de produtos um estudo exploratório em seis setores econômicos. In: FIGUEIREDO, K. F.; FLEURY, P. F.; WANKE, P. (Org.). Logística e gerenciamento da cadeia de suprimentos: planejamento do fluxo de produtos e dos recursos. São Paulo: Atlas, 2003. 
WANKE, P. Gestão de estoques na cadeia de suprimento: Decisões e modelos quantitativos. São Paulo: Atlas, 2011.

YIN, R. K. Estudo de caso: planejamento e métodos. 2. ed. Porto Alegre: Bookman, 2001.

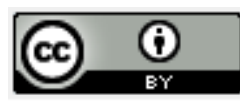

Artigo recebido em 13/12/2015 e aceito para publicação em 01/11/2016

DOI:http://dx.doi.org/ 10.14488/1676-1901.v16i4.2287

Revista Produção Online, Florianópolis, SC, v. 16, n. 4, p. 1262-1284, out./dez. 2016. 